\title{
On Conformal Field Theory of $\operatorname{SLE}(\kappa, \rho)$
}

\author{
Kalle Kytölä \\ kalle.kytola@helsinki.fi \\ Department of Mathematics and Statistics, P.O. Box 68 \\ FIN-00014 University of Helsinki, Finland.
}

\begin{abstract}
$\operatorname{SLE}(\kappa ; \underline{\rho})$, a generalization of chordal Schramm-Löwner evolution (SLE), is discussed from the point of view of statistical mechanics and conformal field theory (CFT). Certain ratios of CFT correlation functions are shown to be martingales. The interpretation is that $\operatorname{SLE}(\kappa ; \underline{\rho})$ describes an interface in a statistical mechanics model whose boundary conditions are created in the Coulomb gas formalism by vertex operators with charges $\alpha_{j}=\frac{\rho_{j}}{2 \sqrt{\kappa}}$. The total charge vanishes and therefore the partition function has a simple product form. We also suggest a generalization of $\operatorname{SLE}(\kappa ; \underline{\rho})$.
\end{abstract}

\section{Introduction}

Schramm-Löwner evolutions (SLEs) are conformally invariant growth processes in two dimensions. From statistical mechanics point of view they describe interfaces in the continuum limits of critical models. The relation of SLEs to the succesful conformal field theory (CFT) approach of physicists has attracted attention since the introduction of SLE. This paper gives a conformal field theory interpretation of a generalization $\operatorname{SLE}(\kappa, \underline{\rho})$ of chordal SLEs, following the approach of e.g. [1, [2, 3], 4]. In a sense the aim is to show how the method of [4] can be applied to this case. A different approach was presented in [5].

The chordal $\operatorname{SLE}(\kappa)$ is known to be in some sense the most general conformally invariant growth process in simply connected regions that only depends on two points on the boundary of the region, the starting point and 
the end point. $\operatorname{SLE}(\kappa ; \rho)$ is the most general growth process depending on three points, the starting point and two other points. For a higher number of boundary points $\operatorname{SLE}(\kappa ; \rho)$ is no longer the most general case. Indeed, in section 3 we find a class of such processes compatible with the CFT representation of statistical physics models. $\operatorname{SLE}(\kappa ; \rho)$ appears as the case where total charge needed in the Coulomb gas formalism is zero and no screening charges are inserted.

Section 2 recalls the definition and some basic properties of $\operatorname{SLE}(\kappa ; \underline{\rho})$. For later application and in order to exhibit the role of infinity, the SLE equation is written in a strip geometry with another normalization of the uniformizing maps. Section 3 contains the main steps of a computation to show that a well chosen ratio of CFT correlation functions becomes a martingale. The interpretation and corresponding results for other SLEs are briefly discussed. An example probability and it's possible meaning in the massless free boson case $\kappa=4$ are the subject of section 4 . Finally, a more algebraic approach (in the spirit of e.g. [6]) to the result of this paper is sketched in section 5 .

\section{SLE basics}

\subsection{Definitions}

Let us give a brief introduction to SLEs and fix some notation. For good general treatments of SLEs the reader may want to see e.g. [7, 8] or [9]. The generalizations $\operatorname{SLE}(\kappa ; \rho)$ and $\operatorname{SLE}(\kappa ; \underline{\rho})$ were introduced in [10] and [11] respectively.

We will recall the definition of $\operatorname{SLE}(\kappa ; \rho)$ in the upper half plane $\mathbb{H}=$ $\{z \in \mathbb{C}: \Im \mathrm{m}(z)>0\}$. The parameters of the process are $\kappa \geq 0$, which corresponds to how the curve looks locally (or the central charge of the CFT), and $\underline{\rho}=\left(\rho_{1}, \ldots, \rho_{n}\right) \in \mathbb{R}^{n}$ which has to do with what kind of boundary conditions are imposed. The initial conditions are the starting point of the curve $\xi_{0} \in \partial \mathbb{H}=\mathbb{R}$ and the locations of the boundary conditions $x_{1}, \ldots, x_{n} \in \mathbb{R}$.

The chordal $\operatorname{SLE}(\kappa, \underline{\rho})$ process in the upper half plane is defined ${ }^{1}$ by the Löwners ordinary differential equation for a uniformizing conformal map

$$
\frac{\mathrm{d}}{\mathrm{d} t} g_{t}(z)=\frac{2}{g_{t}(z)-\xi_{t}}, \quad g_{0}(z)=z \in \mathbb{H},
$$

\footnotetext{
${ }^{1}$ Strictly speaking one first assumes existence of a process $\xi_{t}$ with the desired distribution and then considers the ODE (1).
} 
where $\xi_{t}$, the driving process, is a solution of the following Itô stochastic differential equation

$$
\mathrm{d} \xi_{t}=\sqrt{\kappa} \mathrm{d} B_{t}+\sum_{j=1}^{n} \frac{\rho_{j}}{\xi_{t}-g_{t}\left(x_{j}\right)} \mathrm{d} t
$$

started at $\xi_{0}$ and $\left(B_{t}\right)_{t>0}$ is a standard Brownian motion. The stochastic differential equation (2) has a solution as long as all $\left|\xi_{t}-g_{t}\left(x_{j}\right)\right|$ stay bounded away from zero, i.e. up to the the time $\tau$ of first hitting of $\xi_{t}$ and some $g_{t}\left(x_{j}\right)$. We can define $g_{t}$ by (10) up to the same time. For $z \in \mathbb{H}$ the flow $g_{t}(z)$ is well defined up to the first time $\tau_{z}$ that $g_{t}(z)$ hits $\xi_{t}$, i.e. $\tau_{z}=\inf \{t \in$ $\left.[0, \tau): \liminf _{s \uparrow t}\left|g_{s}(z)-\xi_{s}\right|=0\right\}$. The SLE hull at time $t<\tau$ is defined as $K_{t}:=\left\{z \in \mathbb{H} \mid \tau_{z} \leq t\right\}$. Then $\left(K_{t}\right)_{0 \leq t<\tau}$ is a growing family of hulls, $K_{s} \subset K_{t}$ for $s<t$. The complement $\mathbb{H} \backslash K_{t}$ is simply connected and $g_{t}$ is the unique conformal mapping $\mathbb{H} \backslash K_{t} \rightarrow \mathbb{H}$ with so called hydrodynamic normalization $g_{t}(z)=z+o(1)$ at $z \rightarrow \infty$. The key idea of SLE is to describe the random growth process of hulls $\left(K_{t}\right)_{0 \leq t<\tau}$ in terms of the family of uniformizing maps $\left(g_{t}\right)_{0 \leq t<\tau}$.

The hull grows only locally, at the point $\gamma_{t} \in \overline{\mathbb{H}}$, which is mapped to the driving process, $g_{t}\left(\gamma_{t}\right)=\xi_{t}$. The trace $t \mapsto \gamma_{t}$ is a continuos path almost surely. For $\kappa \leq 4$ the trace is non-self-intersecting and $K_{t}=\gamma_{[0, t]}$. For $4<\kappa<8$ the trace has self-intersections and it "swallows" regions so $\gamma_{[0, t]} \subsetneq K_{t}$. In the parameter range $\kappa \geq 8$ the trace is space filling, $\gamma_{[0, t]}$ has Hausdorff dimension 2.

With $\rho=0 \in \mathbb{R}^{n}$ the process is called the chordal $\operatorname{SLE}(\kappa)$ from $\xi_{0}$ to $\infty$. The driving process is then just a Brownian motion of variance parameter $\kappa$ and it can of course be defined for all $t \geq 0$, that is $\tau=\infty$.

In $[4$ it is shown how some multiple SLEs give rise to $\operatorname{SLE}(\kappa ; \underline{\rho})$ processes. $\operatorname{SLE}(\kappa ; \kappa-6)$ is a chordal $\operatorname{SLE}(\kappa)$ from $\xi_{0}$ to $x_{1}$, but the uniformizing maps $g_{t}$ have a non standard normalization. A special $n \operatorname{SLE}$ gives rise to $\operatorname{SLE}(\kappa ; \underline{\rho})$ with $\rho=(2,2, \ldots, 2) \in \mathbb{R}^{n-1}$.

\section{2 $\operatorname{SLE}(\kappa ; \rho)$ in a strip}

We can cast $\operatorname{SLE}(\kappa ; \rho)$ to a form which treats $x_{1}$ and $\infty$ symmetrically. Assume that $x_{1}<0=\bar{\xi}_{0}$, other cases lead to obvious changes. The conformal mapping $m(z)=-\log \frac{-x_{1}}{z-x_{1}}$ from $\mathbb{H}$ to $\mathbb{S}=\{w \in \mathbb{C}: 0<\Im \mathrm{m}(w)<\pi\}$ maps the boundary points $x_{1}, 0, \infty$ to $-\infty, 0,+\infty$. Thus the family

$$
h_{t}(w)=-\log \frac{\xi_{t}-g_{t}\left(x_{1}\right)}{g_{t}\left(m^{-1}(w)\right)-g_{t}\left(x_{1}\right)}
$$


maps subsets $\mathbb{S} \backslash m\left(K_{t}\right)$ to $\mathbb{S}$, so it encodes the growth of the conformal images of $K_{t}$. The normalization of these conformal maps is such that $h_{t}( \pm \infty)= \pm \infty$ and $h_{t}\left(m\left(\gamma_{t}\right)\right)=0$. A straightforward computation gives

$$
\begin{aligned}
\mathrm{d} h_{t}(w)= & -\sqrt{\kappa} \frac{\mathrm{d} B_{t}}{\xi_{t}-g_{t}\left(x_{1}\right)}+\left(-3+\frac{\kappa}{2}-\rho_{1}-\sum_{j=2}^{n} \frac{\rho_{j}}{2}\right. \\
& \left.+\operatorname{coth}\left(\frac{h_{t}(w)}{2}\right)+\sum_{j=2}^{n} \frac{\rho_{j}}{2} \operatorname{coth}\left(\frac{h_{t}\left(m\left(x_{j}\right)\right)}{2}\right)\right) \frac{\mathrm{d} t}{\left(\xi_{t}-g_{t}\left(x_{1}\right)\right)^{2}} .
\end{aligned}
$$

After a time change $\mathrm{d} t=\left(\xi_{t}-g_{t}\left(x_{1}\right)\right)^{2} \mathrm{~d} s$ and corresponding redefinition $\hat{h}_{s}=h_{t(s)}$ this takes a more convenient form

$$
\begin{aligned}
\mathrm{d} \hat{h}_{s}(w)= & -\sqrt{\kappa} \mathrm{d} B_{s}+\frac{\kappa-6-\sum_{j=1}^{n} \rho_{j}}{2} \mathrm{~d} s \\
& +\sum_{j=1}^{n} \frac{\rho_{j}}{2} \operatorname{coth}\left(\frac{\hat{h}_{s}\left(\tilde{x}_{j}\right)}{2}\right) \mathrm{d} s+\operatorname{coth}\left(\frac{\hat{h}_{s}(w)}{2}\right) \mathrm{d} s,
\end{aligned}
$$

where $\tilde{x}_{j}=m\left(x_{j}\right)$, in particular $\tilde{x}_{1}=-\infty$. This suggests we should define

$$
\rho_{\infty}=\kappa-6-\sum_{j=1}^{n} \rho_{j}
$$

and $\tilde{x}_{\infty}=+\infty=m(\infty) \in \partial \mathbb{S}$. The equation can also be written in terms of

$$
\begin{aligned}
g_{s}^{\mathbb{S}}(w) & =\hat{h}_{s}(w)+\eta_{s} \quad \text { and } \\
\mathrm{d} \eta_{s} & =\sqrt{\kappa} \mathrm{d} B_{s}-\sum_{j \in\{1,2, \ldots, n, \infty\}} \frac{\rho_{j}}{2} \operatorname{coth}\left(\frac{g_{s}^{\mathbb{S}}\left(\tilde{x}_{j}\right)-\eta_{s}}{2}\right) \mathrm{d} s
\end{aligned}
$$

so that $g_{s}^{\mathbb{S}}$ uniformizes the complement of $m\left(K_{t(s)}\right)$ in $\mathbb{S}$ and we have the Löwner equation in the strip

$$
\frac{\mathrm{d}}{\mathrm{d} s} g_{s}^{\mathbb{S}}(w)=\operatorname{coth}\left(\frac{g_{s}^{\mathbb{S}}(w)-\eta_{s}}{2}\right)
$$

From the half plane equations for $\operatorname{SLE}(\kappa ; \underline{\rho})$ it is not immediately clear what kind of boundary condition is imposed at $\infty$. The above coordinate change to the strip $\mathbb{S}$ shows the role of $\infty$. In particular, for any $\operatorname{SLE}(\kappa, \underline{\rho})$, we have $\sum_{j=1}^{n} \rho_{j}+\rho_{\infty}=\kappa-6$, which in section 3 is seen to be related to charge neutrality in the Coulomb gas formalism. 
As an example of the coordinate change we observe that the $\operatorname{SLE}\left(\kappa ; \rho_{1}\right)$ which treats $x_{1}$ and $\infty$ symmetrically is at the value $\rho_{1}=(\kappa-6) / 2$, since only in this case we have $\rho_{1}=\rho_{\infty}$. In the strip geometry this special case is the dipolar SLE with its usual normalization (see e.g. [3] - the drift $\frac{\rho_{1}}{2} \operatorname{coth}(-\infty)+\frac{\rho_{\infty}}{2} \operatorname{coth}(+\infty)$ of the driving process $\eta_{s}$ vanishes.

\section{CFTs of $\operatorname{SLE}(\kappa ; \rho)$}

In this section we find the conformal field theory appropriate for $\operatorname{SLE}(\kappa ; \rho)$. The result is a straightforward application of the ideas of [4] and [3], [2], [1] to the present setup. It turns out that we only need to use boundary primary fields as boundary changing operators at the extra points. Not all such cases are described by $\operatorname{SLE}(\kappa ; \underline{\rho})$, and this leads us to propose a generalization.

\subsection{Statistical mechanics and martingales for SLEs}

We briefly remind the reader of a general argument about how SLEs are related to statistical mechanics. The argument is presented in more detail in [4] and [3].

Different SLEs should represent interfaces in the continuum limits of critical models of statistical mechanics with different boundary conditions. We assume the model is defined in a simply connected domain $D \subset \mathbb{C}$ and we will use a parametrization of the interface by a path $\gamma:[0, \infty) \rightarrow D$. In two dimensions such models have continuum limits desribed by conformal field theories. The expected value of an observale $\mathcal{O}$ then becomes

$$
\mathbb{E}[\mathcal{O}]=\frac{\left\langle\mathcal{O} \mathcal{O}_{\text {b.c. }}\right\rangle_{D}^{\mathrm{CFT}}}{\left\langle\mathcal{O}_{\text {b.c. }}\right\rangle_{D}^{\mathrm{CFT}}}
$$

where $\langle\cdots\rangle_{D}^{\mathrm{CFT}}$ denote conformal field theory correlation functions in the domain $D$ of the model and we have explicitly put operator $\mathcal{O}_{\text {b.c. }}$ which accounts for the boundary conditions of the model (one shouldn't simply take vacuum expected value of $\mathcal{O}$ ). Note that it is also necessary to divide by the correlation function of boundary changes alone, which plays the role of the partition function - otherwise the expected value of the identity operator would differ from unity.

Taking instead conditional expected values conditioned on the knowledge of a portion of the interface $\left.\gamma\right|_{[0, t]}$ defines a martingale (assuming integrability of the random variable $\mathcal{O}$ ). But in many interesting cases the remaining part of the interface arises from the same model defined in a subdomain 
$D_{t} \subset D$, which is essentially the original domain minus the portion of the interface. In such a case

$$
\mathbb{E}\left[\mathcal{O}|\gamma|_{[0, t]}\right]=\frac{\left\langle\mathcal{O} \mathcal{O}_{\text {b.c. }}\left(\gamma_{t}, x_{1}, x_{2}, \ldots\right)\right\rangle_{D_{t}}^{\mathrm{CFT}}}{\left\langle\mathcal{O}_{\text {b.c. }}\left(\gamma_{t}, x_{1}, x_{2}, \ldots\right)\right\rangle_{D_{t}}^{\mathrm{CFT}}}
$$

is a martingale (the boundary condition changes only by the location where the interface continues that is $\gamma_{0}$ is replaced with $\gamma_{t}$ ). Using the transformation properties of CFT operators under conformal mappings we can express this as a ratio of CFT correlation functions in the original domain. SLEs give us explicitly such transformations $g_{t}$ which map certain connected component $D_{t}$ of $D \backslash \gamma_{[0, t]}$ back to the original domain. By conformal covariance one usually chooses $D=\mathbb{H}$ when discussing SLEs.

Several cases involving different SLEs and different boundary conditions have already been studied. The first observation in [1] was that the ordinary chordal SLE corresponds to a theory of central charge $c=c(\kappa)=\frac{(6-\kappa)(3 \kappa-8)}{2 \kappa}$ and a boundary changing operator $\mathcal{O}_{\text {b.c. }}=\Psi_{1,2}(\infty) \Psi_{1,2}(0)$, which creates the two ends of the interface $\gamma$ at 0 and $\infty$. The operator $\Psi_{1,2}$ stands for a boundary primary field of a degenerate conformal weight ${ }^{2} h_{1,2}=\frac{6-\kappa}{2 \kappa}$ and this operator has a vanishing descendant at level 2. In all other cases the central charge is given by the same formula and the interface is again created by $\Psi_{1,2}$. In [2] the radial SLE was worked out with the result $\mathcal{O}_{\text {b.c. }}=\Phi_{0, \frac{1}{2}}\left(z^{*}\right) \Psi_{1,2}(0), z^{*}$ being the endpoint of the interface in the interior of the domain. The dipolar SLE, already seen to be the symmetric case of $\operatorname{SLE}(\kappa ; \rho)$, was studied in 3 and it has boundary conditions $\mathcal{O}_{\text {b.c. }}=\Psi_{0, \frac{1}{2}}\left(x_{-}\right) \Psi_{0, \frac{1}{2}}\left(x_{+}\right) \Psi_{1,2}(0)$. The proposal for multiple SLEs in [4] was taken to include only creations of interfaces on the real axis and something consistent at infinity: $\mathcal{O}_{\text {b.c. }}=\Psi_{h_{\infty}}(\infty) \Psi_{1,2}\left(x_{1}\right) \cdots \Psi_{1,2}\left(x_{n}\right)$. We will soon see that $\operatorname{SLE}(\kappa ; \underline{\rho})$ can be understood with

$$
\mathcal{O}_{\text {b.c. }}=\Psi_{\delta_{\infty}}(\infty) \Psi_{\delta_{1}}\left(x_{1}\right) \cdots \Psi_{\delta_{n}}\left(x_{n}\right) \Psi_{1,2}(0)
$$

again including the creation of the interface at 0 and boundary changes at $x_{1}, \ldots, x_{n}, \infty$. The conformal weights are explicitly

$$
\delta_{j}=\frac{\rho_{j}\left(\rho_{j}+4-\kappa\right)}{4} \kappa \quad \text { for } j=1, \ldots, n, \infty .
$$

\footnotetext{
${ }^{2}$ Here and in the sequel we use the following Kac labeling of the conformal weights

$$
h_{r, s}=\frac{1}{16 \kappa}\left(\kappa^{2}\left(r^{2}-1\right)-8 \kappa(r s-1)+16\left(s^{2}-1\right)\right)
$$

which is convenient in the SLE context. These are the weights for which the Verma module
} is reducible. 
This formula fits nicely to the Coulomb gas formalism (the Coulomb gas formalism of CFT is described in e.g [12] and 13]). To obtain central charge $c=c(\kappa)$ one introduces a background charge $-2 \alpha_{0}=\frac{4-\kappa}{2 \sqrt{\kappa}}$. Then vertex operators of charge $\alpha$ have conformal weight $h(\alpha)=\alpha^{2}-2 \alpha_{0} \alpha=$ $\frac{1}{4 \kappa}(2 \sqrt{\kappa} \alpha)(2 \sqrt{\kappa} \alpha+4-\kappa)$. This means that the conformal weight corresponding to $\rho_{j}$ is obtained with the charge $\alpha_{j}=\frac{\rho_{j}}{2 \sqrt{\kappa}}$. There is, in addition, the operator creating the interface which has charge $\alpha_{1,2}=\frac{1}{\sqrt{\kappa}}$. For $\operatorname{SLE}(\kappa, \underline{\rho})$ the sum of all charges vanishes

$$
-2 \alpha_{0}+\sum_{j \in\{1,2, \ldots, n, \infty\}} \alpha_{j}+\alpha_{1,2}=0
$$

which means that there is no need for screening charges in the Coulomb gas correlation function

$$
\begin{aligned}
& \left\langle V_{\alpha_{\infty}}(\infty) V_{\alpha_{1}}\left(x_{1}\right) \cdots V_{\alpha_{n}}\left(x_{n}\right) V_{\alpha_{1,2}}(\xi)\right\rangle \\
= & \left\langle v_{2 \alpha_{0}-\alpha_{\infty}}^{*}, V_{\alpha_{1}}\left(x_{1}\right) \cdots V_{\alpha_{n}}\left(x_{n}\right) V_{\alpha_{1,2}}(\xi) v_{0}\right\rangle \\
= & \left(\prod_{j=1}^{n}\left(x_{j}-\xi\right)^{\rho_{j} / \kappa}\right)\left(\prod_{1 \leq j<k \leq n}\left(x_{k}-x_{j}\right)^{\rho_{j} \rho_{k} / 2 \kappa}\right) .
\end{aligned}
$$

\subsection{SLE with primary boundary changing operators}

We now consider the question of what kinds of SLEs can arise from creation of one interface with $\Psi_{1,2}$ and with a number of boundary changes implemented by boundary primary fields. All of these "SLEs" seem natural and probably deserve furter study. The $\operatorname{SLE}(\kappa ; \underline{\rho})$ processes are the simplest such processes (in the sense that one doesn't need screening charges) and in this note we concentrate on them.

Take the domain to be the upper half-plane, $D=\mathbb{H}$, and suppose $\mathcal{O}_{\mathrm{b} . \mathrm{c}}=$ $\Psi_{\delta_{\infty}}(\infty) \Psi_{\delta_{n}}\left(x_{n}\right) \cdots \Psi_{\delta_{1}}\left(x_{1}\right) \Psi_{1,2}(0)$. Then the Löwner mapping $g_{t}: H_{t} \rightarrow \mathbb{H}$ with standard time parametrization satisfies $\frac{\mathrm{d}}{\mathrm{d} t} g_{t}(z)=\frac{2}{g_{t}(z)-\xi_{t}}$. Take the driving process to be of the form $\mathrm{d} \xi_{t}=\sqrt{\kappa} \mathrm{d} B_{t}+f \mathrm{~d} t$ so that the interface looks locally like $\operatorname{SLE}(\kappa)$ and we should expect $c=c(\kappa)=\frac{(6-\kappa)(3 \kappa-8)}{2 \kappa}$ again. For simplicity start with an observable which is a product of boundary primary fields, $\mathcal{O}=\Psi_{h_{1}}\left(y_{1}\right) \cdots \Psi_{h_{m}}\left(y_{m}\right)$ with $y_{1}, \ldots, y_{m} \in \mathbb{R}$. From now on all CFT correlation functions will be in $\mathbb{H}$ so we omit the subscript and superscript. 
Applying the conformal transformation $g_{t}$ to (4) we find that the ratio

$$
\frac{\left.\prod_{i=1}^{m} g_{t}^{\prime}\left(y_{i}\right)^{h_{i}}\left\langle\prod_{i=1}^{m} \Psi_{h_{i}}\left(g_{t}\left(y_{i}\right)\right) \Psi_{\delta_{\infty}}(\infty) \prod_{j=1}^{n} \Psi_{\delta_{j}}\left(g_{t}\left(x_{j}\right)\right)\right) \Psi_{1,2}\left(\xi_{t}\right)\right\rangle}{\left\langle\Psi_{\delta_{\infty}}(\infty) \prod_{j=1}^{n} \Psi_{\delta_{j}}\left(g_{t}\left(x_{j}\right)\right) \Psi_{1,2}\left(\xi_{t}\right)\right\rangle}
$$

should be a local martingale. Let us denote the numerator by $N_{t}$ and denominator by $D_{t}$.

The following computation is similar to one in 4 so only main steps are given here. Making use of the null field $\left(-2 \mathcal{L}_{-2}+\frac{\kappa}{2} \mathcal{L}_{-1}^{2}\right) \Psi_{1,2}$ and Ward identity we compute the Itô derivatives

$$
\begin{gathered}
\mathrm{d} N_{t}=\mathcal{D} N_{t} \quad \mathrm{~d} D_{t}=\mathcal{D} D_{t} \\
\mathcal{D}=\mathrm{d} t\left(\sum_{j=1}^{n} \frac{2 \delta_{j}}{\left(\xi-X^{(j)}\right)^{2}}+f \partial_{\xi}\right)+\mathrm{d} B_{t}\left(\sqrt{\kappa} \partial_{\xi}\right) .
\end{gathered}
$$

The Itô derivative of the ratio $N_{t} / D_{t}$ should have no drift

$$
\mathrm{d}\left(\frac{N_{t}}{D_{t}}\right)=\left(f-\kappa \frac{\partial_{\xi} D}{D}\right) \partial_{\xi}\left(\frac{N_{t}}{D_{t}}\right) \mathrm{d} t+\sqrt{\kappa} \partial_{\xi}\left(\frac{N_{t}}{D_{t}}\right) \mathrm{d} B_{t},
$$

which leads to the requirement $f=\kappa \partial_{\xi} \log D_{t}$.

The above requirement gives a class of interesting processes generalizing $\operatorname{SLE}(\kappa ; \rho)$. One could start with any conformal block for the correlation function $D=\left\langle\Psi_{\delta_{\infty}}(\infty) \prod_{j=1}^{n} \Psi_{\delta_{j}}\left(x_{j}\right) \Psi_{1,2}(\xi)\right\rangle$ and define the driving process $\xi_{t}$ by

$$
\mathrm{d} \xi_{t}=\sqrt{\kappa} \mathrm{d} B_{t}+\kappa\left(\partial_{\xi} \log D\left(\xi_{t} ; g_{t}\left(x_{1}\right), \ldots, g_{t}\left(x_{n}\right)\right)\right) \mathrm{d} t .
$$

Here we are content to remark that with conformal weights $\delta_{j}$ given by (5), as a consequence of the charge neutrality the Coulomb gas correlation function (6) provides us the nice factorizable $D\left(\xi ; x_{1}, \ldots, x_{n}\right)$. This choice of $D$ gives rise to the drift term of $\operatorname{SLE}(\kappa ; \underline{\rho})$

$$
f=\kappa \partial_{\xi} \log \left\langle V_{\alpha_{\infty}}(\infty) V_{\alpha_{1}}\left(x_{1}\right) \cdots V_{\alpha_{n}}\left(x_{n}\right) V_{\alpha_{1,2}}(\xi)\right\rangle=\sum_{j=1}^{n} \frac{\rho_{j}}{\xi-x_{j}} .
$$

Note that $f \sim \rho_{j} /\left(\xi-x_{j}\right)$ fixes the asymptotic behavior of $D$ as $\xi \rightarrow x_{j}$ and fusion rule then allows no other conformal weight of a primary field at $x_{j}$ than (15).

Concerning the generalization of $\operatorname{SLE}(\kappa ; \underline{\rho})$ suggested above and computations in SLE in general we also emphasise that even if the conformal weight of some boundary changing operator coincides with a weight $h_{r, s}$, the operator may or may not have a vanishing descendant. Such phenomenon is not new in the SLE context, see e.g. 1] and [14. 


\section{$4 \quad$ Free field and $\operatorname{SLE}(4 ; \rho)$}

At $\kappa=4$ the conformal field theory has central charge $c=1$ and SLE should correspond to "level sets" of the massless free boson field ${ }^{3}$. The next sections give a few indications of how $\operatorname{SLE}(\kappa ; \underline{\rho})$ fits into the picture.

The application of $\operatorname{SLE}(\kappa ; \underline{\rho})$ to the free field was considered in $[\underline{5]}$. The physics was discussed in more depth, but let us for illustration purposes state a conclusion from there. Let the free field $\varphi$ have piecewise constant Dirichlet boundary conditions with jumps at $\xi_{0}, x_{1}, \ldots, x_{n}$. The jump at $\xi_{0}$ has the critical value $\lambda^{*}=\frac{1}{\sqrt{4 g}}$, such that we can trace the discontinuity line of this jump. At $x_{j}$ one can take a jump of any size $\lambda_{j}$. The conformal weight of the corresponding operator is $\delta_{j}=g \lambda_{j}^{2}$, in particular for the critical jump size this is $\frac{1}{4}$. The partition function for such a free field is

$$
Z \sim\left(\prod_{j=1}^{n}\left(x_{j}-\xi\right)^{\sqrt{g} \lambda_{j}}\right)\left(\prod_{1 \leq j<k \leq n}\left(x_{k}-x_{j}\right)^{2 g \lambda_{k} \lambda_{j}}\right)
$$

of the form (6) that corresponds to $\operatorname{SLE}(\kappa ; \underline{\rho})$. In $[5]$ there is also a discussion that points to the interpretation given next.

\subsection{Probability to be on the left of the trace}

In this section we compute a certain example probability for $\operatorname{SLE}(\kappa, \rho)$ with $\kappa>4$ and observe that its limit at $\kappa=4$ has a natural interpretation in terms of free field boundary conditions. Computation of this probability for the dipolar SLE appeared in 3].

Recall from section 2.2 that the $\operatorname{SLE}(\kappa ; \rho)$ equation in $\mathbb{S}$ is conveniently written in terms of the mapping $\hat{h}_{s}$ which maps the tip of SLE trace to 0

$$
\mathrm{d} \hat{h}_{s}(w)=-\sqrt{\kappa} \mathrm{d} B_{s}+\frac{\kappa-6-2 \rho}{2} \mathrm{~d} s+\operatorname{coth}\left(\frac{\hat{h}_{s}(w)}{2}\right) \mathrm{d} s .
$$

Suppose we have an analytic function $F: \mathbb{S} \rightarrow \mathbb{C}$ such that $F\left(\hat{h}_{s}(w)\right)$ is a $\mathbb{C}$-valued martingale. The drift of $F\left(\hat{h}_{s}\right)$ should vanish, which by Itô's formula means

$$
0=\frac{\kappa-6-2 \rho}{2} F^{\prime}(\hat{h})+\operatorname{coth}\left(\frac{\hat{h}}{2}\right) F^{\prime}(\hat{h})+\frac{\kappa}{2} F^{\prime \prime}(\hat{h}) .
$$

\footnotetext{
${ }^{3}$ There is yet unpublished work by Scott Sheffield and Oded Schramm which shows that chordal SLE(4) is a discontinuity curve between two levels.
} 
Conversely, the solutions to this equation give local martingales. Constants solve the equation, but the other linearly independent solution is an integral function of

$$
(\sinh (u / 2))^{-4 / \kappa} \exp \left(\frac{6-\kappa+2 \rho}{\kappa} u\right) .
$$

The real and imaginary parts of that analytic function are harmonic local martingales for $\operatorname{SLE}(\kappa ; \rho)$ in $\mathbb{S}$.

We assume that $(\kappa-8) / 2<\rho<(\kappa-4) / 2$ so that the integral function is finite at $\pm \infty$ and that $\kappa>4$ so that it is finite at 0 . Then the integral function can be taken to be

$$
F(w)=\int_{-\infty}^{w}(\sinh (u / 2))^{-4 / \kappa} \exp \left(\frac{6-\kappa+2 \rho}{\kappa} u\right) \mathrm{d} u .
$$

Let us set

$$
P_{\kappa ; \rho}^{l}(w)=1-\frac{\Im m F(w)}{\Im m F(+\infty)},
$$

which takes the value 1 at $-\infty$ and 0 on the positive real axis. If a point $w \in \mathbb{S}$ is swallowed, we have $\lim _{s \uparrow \sigma_{w}} \hat{h}_{s}(w)=0$ where $\sigma_{w}$ is the explosion time of $w$ for the Löwner equation in the strip. If $w$ is on the left (resp. right) of the hull, we have $\lim _{s \uparrow \sigma_{w}} \hat{h}_{s}(w)=-\infty$ (resp. $+\infty$ ). Thus the function $P_{\kappa ; \rho}^{l}(w)$ gives the probability of $w$ being on the left of the hull, $P_{\kappa ; \rho}^{l}\left(\hat{h}_{s}(w)\right)=\mathbb{E}\left[\mathbf{1}_{w \text { on left }}|\hat{\gamma}|_{[0, s]}\right]$.

\subsection{Free field boundary conditions}

As $\kappa \downarrow 4$ the bounded harmonic function $P_{\kappa ; \rho}^{l}(w)$ has a limit $p_{\rho}^{l}(w)$, which is a harmonic $\operatorname{SLE}(4 ; \rho)$-martingale. It's boundary conditions are 0 on the positive real axis and 1 on the negative real axis, so it describes the probability that $w$ will be on the left of the trace at the time the trace hits the the upper boundary of the strip. On the upper boundary $w=x+i \pi$ the directional derivative of $p_{\rho}^{l}$ to direction $e^{-i \rho \pi / 2}$ vanishes.

If $\operatorname{SLE}(4 ; \rho)$ describes a "level set" of the free field, then the boundary condition on the upper boundary seems to correspond to the free field $\varphi$ having a vanishing directional derivative to the direction $e^{-i \rho \pi / 2}$. The computation only made sense if $-2<\rho<0$ whereas the piecewise constant Dirichlet boundary conditions of [5] don't have such restriction.

The boundary changing operator at 0 has the dimension $\frac{1}{4}$ and it changes boundary condition between two different Dirichlet boundaries. The operators at $-\infty,+\infty$ have dimensions $\frac{\rho^{2}}{16}, \frac{\rho_{\infty}^{2}}{16}$ and they change the direction of 
the vanishing derivative by an angle $\frac{\pi}{2} \rho, \frac{\pi}{2} \rho_{\infty}$ respectively. The total change of angle is $\frac{\pi}{2}\left(\rho+\rho_{\infty}\right)=-\pi$ which is consistent with returning to Dirichlet boundary condition. The particularly interesting case $\rho=-1$ (the dipolar SLE) corresponds to Neumann boundary condition as discussed in 3 .

For a general $\operatorname{SLE}(4 ; \underline{\rho})$ we always have a boundary changing operator $\Psi_{1,2}$ of dimension $\frac{1}{4}$ at $\xi$ so the curve is created by a jump of critical size $\lambda^{*}$ in the Dirichlet boundary conditions. The other boundary changing operators have dimensions $\frac{1}{16} \rho_{j}^{2}$ such that $\sum_{j \in\{1, \ldots, n, \infty\}} \frac{\pi}{2} \rho_{j}=-\pi$.

\section{Virasoro module valued martingale}

The approach of section 3 as well as of 4 is slightly different from that of [1], 2], 6], 3]. We have shown that certain CFT correlation functions are martingales for SLEs.

A more algebraic approach is to encode the state of the SLE as a vector in a highest weight module for the Virasoro algebra (the CFT Hilbert space should consist of highest weight representations of the Virasoro algebra with a common central charge $c(\kappa))$. We think of starting from the vacuum $|\Omega\rangle$ in an irreducible module of highest weight 0 and then applying intertwining operators $\Psi_{1,2}\left(\xi_{t}\right)$ and $\Psi_{\delta_{j}}\left(g_{t}\left(x_{j}\right)\right)$ to create the appropriate boundary conditions in $\mathbb{H}$. Then we apply an operator $G_{t}$ which implements the conformal transformation $g_{t}^{-1}$ in space of states.

In the next section we will give main steps of computation to show that the process

$$
\left|M_{t}\right\rangle=\frac{1}{D\left(\xi_{t} ; g_{t}\left(x_{1}\right), \ldots\right)} G_{t} \Psi_{\delta_{n}}\left(g_{t}\left(x_{n}\right)\right) \cdots \Psi_{\delta_{1}}\left(g_{t}\left(x_{1}\right)\right) \Psi_{1,2}\left(\xi_{t}\right)|\Omega\rangle
$$

in a highest weight module for the Virasoro algebra is a (vector valued) martingale. Correlation functions of section [3 can be written as $\left\langle u \mid M_{t}\right\rangle$ for some constant vectors $\langle u|$ in the dual of the module. The example of section 4 corresponds to insertion of bulk primary field of dimension 0 at $z=m^{-1}(w) \in \mathbb{H}$, that is $\langle u|=\left\langle\delta_{\infty}\right| \Phi_{0}(z)$.

\subsection{The computation of $\mathrm{d}\left|M_{t}\right\rangle$}

We left the computation of the Itô derivative of $\left|M_{t}\right\rangle$ to this section. The drift will be shown to be zero so that $\left|M_{t}\right\rangle$ is local martingale with values in a module for the Virasoro algebra. There are three kinds of cancellations in the drift term. Some cancellations reflect the fact that $G_{t}$ implements the 
transformation $g_{t}^{-1}$. For a cancellation of another kind it is again crucial to have a null descendant of the intertwining operator $\Psi_{1,2}$ which creates the interface. Finally, it is important to take into account the change of the partition function - the third cancellations arise from the correct choice of the denominator $D\left(\xi ; x_{1}, \ldots, x_{n}\right)$, see section 3 ,

Construction of the operator $G_{t}$, which implements the conformal transformation $g_{t}^{-1}$ of SLE, was treated in [6]. From the Löwner equation (11) it follows that

$$
\mathrm{d} G_{t}=G_{t} e^{\xi_{t} L_{-1}}\left(-2 L_{-2}\right) e^{-\xi_{t} L_{-1}} \mathrm{~d} t .
$$

A boundary intertwining operator $\Psi_{\delta}$ of conformal weight $\delta$ is a family of linear mappings between Virasoro modules $M^{(0)}$ and $M^{(\infty)}$ parametrized by a boundary point $x \in \partial \mathbb{H}=\mathbb{R}$ such that the following intertwining relation holds

$$
\left[L_{n}, \Psi_{\delta}(x)\right]=\left(x^{1+n} \partial_{x}+(1+n) \delta x^{n}\right) \Psi_{\delta}(x)
$$

This means in particular that $L_{-1}$ generates translations,

$$
e^{t L_{-1}} \Psi_{\delta}(x) e^{-t L_{-1}}=\Psi_{\delta}(x+t) .
$$

The most useful form of the intertwining relation for the present setup is

$$
\left[e^{t L_{-1}} L_{n} e^{-t L_{-1}}, \Psi_{\delta}(x)\right]=\left((x-t)^{1+n} \partial_{x}+(1+n) \delta(x-t)^{n}\right) \Psi_{\delta}(x) .
$$

We assume that $M^{(0)}=M_{1,1}$ is the irreducible module of highest weight $h_{1,1}=0, M^{(1)}=M_{1,2}$ is the irreducible module of highest weight $h_{1,2}=$ $\frac{6-\kappa}{2 \kappa}$ and $M^{(2)}, \ldots, M^{(n)}, M^{(n+1)}$ are some highest weight modules for the Virasoro algebra. Let $\Psi_{1,2}(x): M^{(0)} \rightarrow M^{(1)}$ and $\Psi_{\delta_{j}}: M^{(j)} \rightarrow M^{(j+1)}$ for $j=1, \ldots, n$ be intertwining operators of conformal weights $h_{1,2}$ and $\delta_{j}$.

In order to reduce the notation denote $g_{t}\left(x_{j}\right)=X_{t}^{(j)}$ and $\frac{\partial}{\partial X_{t}^{(j)}}=\partial^{(j)}$ and $f=\sum_{j=1}^{n} \frac{\rho_{j}}{\xi_{t}-X_{t}^{(j)}}$. Let us first compute

$$
\begin{aligned}
& \mathrm{d}\left(D\left(\xi_{t} ; X_{t}^{(1)}, \ldots, X_{t}^{(n)}\right)\left|M_{t}\right\rangle\right) \\
= & G_{t} e^{\xi L_{-1}}\left(-\mathrm{d} t 2 L_{-2}\right) e^{-\xi L_{-1}} \prod_{j} \Psi_{\delta_{j}}\left(X_{t}^{(j)}\right) \Psi_{1,2}(\xi)|\Omega\rangle \\
& +G_{t}\left(\mathrm{~d} t \sum_{j=1}^{n} \frac{2}{X_{t}^{(k)}-\xi} \partial^{(k)}\right) \prod_{j} \Psi_{\delta_{j}}\left(X_{t}^{(j)}\right) \Psi_{1,2}(\xi)|\Omega\rangle \\
& +G_{t} \prod_{j} \Psi_{\delta_{j}}\left(X_{t}^{(j)}\right)\left(\mathrm{d} B_{t} \sqrt{\kappa} \partial_{\xi}+\mathrm{d} t f \partial_{\xi}+\mathrm{d} t \frac{\kappa}{2} \partial_{\xi}^{2}\right) \Psi_{1,2}(\xi)|\Omega\rangle .
\end{aligned}
$$


Commute $e^{\xi L_{-1}} L_{-2} e^{-\xi L_{-1}}$ in the first term to the right and observe the cancellations with the second term. Using the null vector

$$
\left(-2 L_{-2}+\frac{\kappa}{2} L_{-1}^{2}\right)\left|h_{1,2}\right\rangle=0
$$

in the form

$$
e^{\xi L_{-1}}\left(-2 L_{-2}+\frac{\kappa}{2} \partial_{\xi}^{2}\right) e^{-\xi L_{-1}} \Psi_{1,2}(\xi)|\Omega\rangle=0
$$

we have another cancellation in the first and third terms. The rest can be written as

$$
\mathrm{d}\left(D\left(\xi_{t} ; X_{t}^{(1)}, \ldots, X_{t}^{(n)}\right)\left|M_{t}\right\rangle\right)=\mathcal{D}\left(D\left(\xi_{t} ; X_{t}^{(1)}, \ldots, X_{t}^{(n)}\right)\left|M_{t}\right\rangle\right),
$$

where $\mathcal{D}$ is as in (7). Using again the facts $\mathrm{d} D_{t}=\mathcal{D} D_{t}$ and $f=\kappa \partial_{\xi} \log D_{t}$, as in section 3, we see that the drift of $\left|M_{t}\right\rangle$ vanishes.

\section{Conclusions}

We exhibited the conformal field theory appropriate for $\operatorname{SLE}(\kappa ; \underline{\rho})$ in the statistical mechanics interpretation of $[4$ and 3 . The result turned out to be particularly simple in the Coulomb gas formalism. In the course of study we proposed other generalizations of $\operatorname{SLE}(\kappa)$ to the case where boundary conditions depend on more than three boundary points.

Acknowledgements: The author is grateful to Michel Bauer and Denis Bernard for interesting discussions on SLEs and conformal field theory and to the anonymous referees for useful suggestions.

\section{References}

[1] M. Bauer and D. Bernard, "Conformal field theories of stochastic Loewner evolutions" Commun. Math. Phys. 239 (2003) 493 arXiv:hep-th/0210015.

[2] M. Bauer and D. Bernard, "CFTs of SLEs: The radial case" Phys. Lett. B 583 (2004) 324 arXiv:math-ph/0310032.

[3] M. Bauer, D. Bernard and J. Houdayer, J. Stat. Mech. 0503 (2005) P001 arXiv:math-ph/0411038. 
[4] M. Bauer, D. Bernard and K. Kytola, "Multiple Schramm-Loewner Evolutions and Statistical Mechanics Martingales" J. Stat. Phys. Vol. 120 (2005) Nos. 5/6 1125-1163 arXiv:math-ph/0503024.

[5] J. Cardy, "SLE(kappa, rho) and conformal field theory" arXiv:math-ph/0412033.

[6] M. Bauer and D. Bernard, "Conformal transformations and the SLE partition function martingale" Annales Henri Poincare 5 (2004) 289 arXiv:math-ph/0305061.

[7] W. Werner, "Random planar curves and Schramm-Loewner evolutions" In Lectures on probability theory and statistics, vol. 1840 of Lecture Notes in Math., p. 107-195. arXiv:math.PR/0303354

Springer, Berlin, 2004.

[8] W. Kager and B. Nienhuis, "A Guide to Stochastic Loewner Evolution and its Applications," arXiv:math-ph/0312056.

[9] J. Cardy, "SLE for theoretical physicists," Annals Phys. 318 (2005) 81 arXiv:cond-mat/0503313.

[10] G. Lawler, O. Schramm, W. Werner, "Conformal restriction. The chordal case" J. Amer. Math. Soc. vol. 16, no. 4, 917-955, 2003 arXiv:math.PR/0209343.

[11] J. Dubédat, "SLE $(\kappa, \rho)$ martingales and duality" Ann. Probab. 2005, vol. 33, no. 1, 223-243 arXiv:math.PR/0303128.

[12] P. Di Francesco, P. Mathieu, D. Sénéchal: "Conformal Field Theory" Springer-Verlag New York, Inc., 1997

[13] G. Felder, "BRST Approach To Minimal Models" Nucl. Phys. B 317 (1989) 215 [Erratum-ibid. B 324 (1989) 548].

[14] M. Bauer and D. Bernard, "SLE, CFT and zig-zag probabilities" in Proceedings of the conference 'Conformal Invariance and Random Spatial Processes', Edinburgh (2003), arXiv:math-ph/0401019. 